\title{
La evaluación psicológica en los campos de la psicología jurídica en Colombia: psicología forense*
}

\section{The psychological evaluation in the fields of legal psychology in Colombia: forensic psychology}

\author{
Nancy M. Vargas Espinosa** \\ ORCID: 0000-0001-9492-9176 \\ Ervyn Hermilzon Norza Cespedes \\ ORCID:0000-0002-6665-0116 \\ Leidy Johana Amaya \\ Lina María Cárdenas \\ Eliana Catalina Delgado \\ Karen Johanna González \\ Lina Marcela Henao \\ Diana Carolina Medina \\ Karen Melissa Pinzón \\ Manuel Felipe Zamora \\ Universidad El Bosque, Colombia \\ Recibido: 22 de octubre de 2018 \\ Revisado: 20 de enero de 2019 \\ Aceptado: 4 de febrero de 2019
}

\section{Resumen}

En este estudio se buscó analizar los procesos de evaluación en el campo de la psicología forense en Colombia, teniendo como producto argumentativo un marco teórico acerca de la definición de la Psicología Forense (funciones y rol), sus problemas éticos, su aplicación en los diferentes campos del derecho, y sus contraflujos más recurrentes como el tipo de instrumentos y entrevistas que utiliza el psicólogo. De acuerdo con lo anterior y teniendo en cuenta los criterios de selección de los peritos para las pruebas, se diseñó una entrevista estructurada aplicada a una muestra de psicólogos del campo forense a nivel nacional realizando un análisis de corte cuantitativo sobre la experiencia del psicólogo forense, su participación en los diferentes clases de perito, las técnicas y criterios que usa como soporte en cualquier proceso, y por último, algunas conclusiones acerca del posicionamiento en que se encuentra el psicólogo forense en Colombia.

Palabras clave: psicología forense, evaluación psicológica, administración de justicia, peritación, perito, ética.

Artículo de Investigación. Cómo citar: Vargas, E. N. M., Norza, C. E. H., Amaya, L. J., Cárdenas, L. M., Delgado, E. C., ... Zamora, M. F. (2019). La evaluación psicológica en los campos de la Psicología Jurídica en Colombia: Psicología Forense. Diversitas: Perspectivas en Psicología, 15(2), 315-333. DOI: $10.15332 / 22563067.5403$

** Correspondencia: Nancy M. Vargas Espinosa. Facultad de Psicología, Universidad El Bosque. Dirección postal: Kr. 9 n. ${ }^{\circ} 11 \mathrm{~A}-12$. Correo electrónico: vargasnancye@unbosque.edu.co 
Nancy M. Vargas Espinosa, Ervyn Hermilzon Norza Cespedes, Leidy Johana Amaya, Lina María Cárdenas, Eliana Catalina Delgado, Karen Johanna González, Lina Marcela Henao, Diana Carolina Medina, Karen Melissa Pinzón, Manuel Felipe Zamora

\section{Abstract}

In this study, we sought to analyze the evaluation processes in the field of forensic psychology in Colombia, having as argumentative product a theoretical framework about the definition of forensic psychology (functions and role), its ethical problems, its application in different fields of law, and its most recurrent counter-flows as the type of instruments and interviews used by the psychologist. In accordance with the foregoing and taking into account the selection criteria of the experts for the tests, a structured interview was designed applied to a sample of forensic field psychologists at a national level performing a quantitative analysis on the experience of the forensic psychologist, their participation in the different types of expert works, the techniques and criteria used as support in any process, and finally, some conclusions about the position in which the forensic psychologist is in Colombia.

Keywords: forensic psychology, psychological evaluation, administration of justice, expert appraisal, expert, ethics.

\section{Introducción}

La psicología forense es un campo reciente en Colombia que al compararlo con los países de la región, ha avanzado en las últimas dos décadas con mayor celeridad y se observan prácticas aceptadas al interior de la praxis de los psicólogos y el sistema judicial, llegando a ser indispensable para dar respuesta a diversos problemas del derecho donde se evidencia la participación particular de protocolos, técnicas y medios científicos de la psicología forense al interior del contexto jurídico (Vargas, Rodríguez \& Norza, 2017; Lin Ching Céspedes, 2002).

Partiendo del desarrollo psico-forense en Colombia, este artículo se planteó como objetivo analizar los procesos de evaluación del campo psicológico forense en Colombia. Así, se realiza inicialmente una contextualización del concepto de psicología forense, ámbitos de uso, tipo de instrumentos y característica de la entrevista que usa este campo, para luego a través de un estudio exploratorio con una muestra de psicólogos forenses a quienes se aplicó una entrevista estructurada, se describen y analizan los resultados que permiten identificar un panorama actual en su ejercicio.

La psicología forense es la ciencia que aplica todas las ramas de la Psicología con el propósito de cooperar con la administración de justicia. Según Morales y García (2010), Rodríguez (2010) y Fernández (2007) (citados por De la Calle, Gómez, Muñoz \& Vásquez, 2012) la Psicología Forense valora y aporta pruebas respecto al comportamiento de un sujeto ceñido por principios científicos que requiere de la formulación y contraste de hipótesis contribuyendo a la correcta administración de la justicia y la toma de decisiones en este ámbito. De acuerdo con lo anterior, se puede decir que esta subárea se fundamenta en la aplicación de métodos y conocimientos para la realización de pruebas periciales en el ámbito del Derecho.

En cuanto a los órganos jurisdiccionales Muñoz y Manzanero (2011), argumentan que la Psicología Forense se aplica, por ejemplo, en los juzgados de familia en donde interviene en procesos de guarda y custodia de los niños, procesos relacionados con el desarrollo del régimen de visitas, impugnación de tutelas, acogimiento familiar, procesos sobre adopción, privación de la patria potestad, nulidad civil del matrimonio y procesos de emancipación de menores de edad. Por otro lado, en los juzgados penales, la Psicología Forense responde a dudas relacionadas con la valoración pericial psicológica del denunciado, entre otras, la capacidad procesal, la imputabilidad y el riesgo delictivo. Por otra parte, se encargan de la valoración pericial psicológica de la víctima, lesiones o secuelas psíquicas, credibilidad del testimonio en abuso sexual infantil, y finalmente, en los procesos de violencia contra la mujer y menores víctimas. En materia civil apoyan los procesos de guarda y custodia, régimen de visitas, acogimiento familiar, procesos de adopción, privación de patria potestad y nulidad civil del matrimonio. 
En cuanto a los menores de edad infractores, se realiza la valoración de la situación psicosocial del menor para orientar respecto a la medida socioeducativa más adecuada, conciliación y reparación, seguimiento de las medidas post-sentencia y supervisión de permisos y cambios de medida, por mencionar algunos (Muñoz \& Manzanero 2011). La Psicología siempre ha estado estrechamente relacionada con la Psicología Criminológica y la Psicología Social (Céspedes, 2002). Por otro lado, Crespi (1994, citado por Morales \& García, 2010), agrega otras funciones de la Psicología Forense, entre otras, la intervención en poblaciones que deben participar en programas de tratamiento, consultoría aplicada a diferentes instancias judiciales, como pueden ser la policía, los abogados, el personal en las cárceles, la respuesta a las inquietudes o dudas que exista en relación al comportamiento humano y particularmente de las personas intervinientes, víctimas, testigos y jueces.

Por tanto, el psicólogo forense debe ser un profesional especializado en su área y estar actualizado en conocimientos científicos tanto en el área de la psicología como en los procesos judiciales, civiles o penales en los que participa (Pinto, Corvalan \& Acuña, citados por Rodríguez, 2010; Hernández \& Tapias, 2010).

Finol y Piña (2008) agregan que, en consecuencia, un psicólogo forense realiza un peritaje psico-legal o informe psicológico, cumpliendo como función una declaración de conocimiento, técnica o practica sobre los hechos enjuiciados, con la finalidad de tomar las mejores decisiones judiciales. Teniendo en cuenta este contexto, el profesional se puede ver expuesto a diferentes demandas que derivan en problemas éticos y deontológicos, e incluso, es en esta área en la que se evidencian conductas que en la praxis de algunos psicólogos pueden ser consideradas negligentes o no éticas. Las normas éticas establecidas para los psicólogos forenses están relacionadas con las competencias que incluyen la adaptación de todas las normas legales y la prioridad de un cocimiento verdadero y apropiado, la sustentación teórica correcta, veracidad y sinceridad en su testimonio y en su informe, claridad de los roles (Molina, 2011). En Colombia el código de procedimiento penal, Ley 609 de 2004, determina los criterios básicos para desempeñarse como perito o testigo experto consignados en los Artículos: 278, 406, 408, 410, 413, 417, 420, 422; además esta Ley describe algunos conceptos, situaciones y procedimientos que competen al saber de la psicología (Art. 252, 253, 267, 271,304, 306, 308, 311) como lo concerniente a grabaciones (audio y videos) como prueba, papel del perito en la defensa, circunstancias de participación y procedimiento.

De acuerdo con Frías (2007), el denominado perito es quien deberá responder de manera concreta y acertada a la pregunta específica relacionada con el interés jurídico, sin ofrendar información innecesaria que llegue a desviar el objeto primario de interés, más que una prueba, este informe representa un medio para la valoración, proporcionando más elementos para la formulación de juicios (Simões, 2011).

De ese modo, en cada uno de los ámbitos del derecho, el psicólogo forense actúa como perito o como asesor, y es considerado auxiliar de la justicia, por ejemplo, el psicólogo forense se puede encargar de la evaluación de los hechos a fin de generar un informe, con el que el juez determinara la inimputabilidad (Finol \& Piña, 2008). La solicitud de dicho dictamen pericial puede ser requerido por jueces, fiscales y abogados, para conocer posibles alteraciones mentales de los autores de delitos, también de la predicción del riesgo de violencia futura en personas que han cometido un delito violento, la credibilidad del testimonio en víctimas de abuso sexual infantil o el daño psicológico en víctimas de delitos violentos. En el ámbito del derecho penal o familiar otra problemática que involucra un psicólogo forense es el maltrato hacia la mujer, la interdicción de derechos la cual hace referencia a la situación de una persona sometida a una tutela, ya que se encuentra en un estado habitual de defecto intelectual que la hace incapaz de proveer sus propios intereses (Echeburúa, Muñoz \& Loinaz 2011; Goldman, s. f.; Natenson, 2007; Ochoa, 2006; Roldán \& Solano, 2001). Por otra parte, es de importancia del psicólogo jurídico la identificación de los simuladores o detección de engaños (Inda, Lemos, López \& Alonso, 2005). Además, los psicólogos tienen como función adicional el asesorar a otros profesionales sobre la veracidad frente a las claves conductuales y el engaño (Masip, 2005). 
Por lo anterior el trabajo del psicólogo forense a la hora de realizar una evaluación de testimonio debe ser muy exhaustivo (Davoglio \& De Lima, 2010). En algunos ámbitos la totalidad de actividad que realiza un psicólogo es la evaluación psicológica tal como lo afirma Buela, Sierra, Carretero y de los Santos (2002). Por otra parte, es importante la reducción de la sobrevaloración y el mal uso de las etiquetas diagnósticas, ya que se pretende un abordaje de tipo descriptivo y funcional (Echeburúa et al., 2011).

Igualmente, Soria (2006) argumenta que los instrumentos no son los únicos recursos que utiliza la psicología forense para evaluar, también se cuenta con la entrevista, la cual ayuda a recaudar información relevante y según Saborío (2005) es una herramienta que ayuda a la corroboración de las evaluaciones y consolidar la validez diagnóstica.

Respecto a la entrevista Rubio (2010) la define como "un proceso en el que intervienen dos o más personas, a través de un medio generalmente oral, en el que se distinguen roles asimétricos: entrevistador-entrevistado" (p.99). Particularmente en el ámbito forense esta debe permitir recolectar datos para sustentar el informe pericial, formular y contrastar hipótesis (Collado, 2009; Rubio, 2010). Para lo cual se cuenta con dos tipos de entrevistas (estructuradas y semi-estructuradas) que le permitirán al psicólogo obtener información detallada acerca de los hechos importantes que serán útiles en el proceso jurídico.

No obstante, aunque la entrevista sea el método más utilizado en este proceso, cabe mencionar que no es totalmente confiable, como lo afirma Amor, Corral y Echeburúa (2003) ya que carece de procesos de estandarización. Sin embargo, de acuerdo con Sierra, Jimenez y Bunce (2006), la entrevista clínica estructurada es una de las herramientas más completas en el campo forense, en este sentido se puede mencionar el SCID I (Structured Clinical Interview for DSM-IV), que es una entrevista semiestructurada que permite establecer los diagnósticos más importantes del eje I del DSM-IV, también permite recaudar información acerca del eje IV y V. Por otro lado, se encuentra el SCID II la cual permite evaluar los diez trastornos de personalidad del eje II del DSM-IV. Además, se incluye un cuestionario de personalidad auto cumplimentado con el fin de indagar en ítems que se han contestado afirmativamente. Por otra parte, se cuenta con la entrevista cognitiva cuyo objetivo es mejorar el recuerdo de la víctima o testigo sobre un acontecimiento, una situación, un delito o una persona (Amar \& Aristizábal, 2012).

Existen otros instrumentos que son de gran uso en el campo forense como el SVR-20 (Sexual Violence Risk) que es una escala para la valoración de riesgo de violencia sexual y se divide en tres secciones, indagando acerca de la desviación sexual del individuo, los antecedentes individuales de delitos $y / 0$ agresiones sexuales y ausencia de planes a futuro viables. Además, permite valorar el riesgo de violencia sexual en pacientes mentales y delincuentes adultos acusados de este tipo de delitos (AndrésPueyo \& Echeburúa, 2010; Tapias-Saldaña, 2011)

El HCR-20 (Historical Clinical Risk -20) o guía para la valoración del riesgo de comportamientos violentos evalúa, según Andrés-Pueyo y Echeburúa (2010) conductas violentas en pacientes mentales y delincuentes adultos. Otra escala conocida y utilizada es el PCL-R (The Hare Psychopathy Checklist-Revised) evalúa la presencia de psicopatía en adultos con un historial violento o delictivo. Este instrumento examina la violencia interpersonal específica. y recoge los datos socios demográficos relacionados con el posible uso de sustancias psicoactivas, los vínculos familiares, entre otros. Cuenta con excelentes niveles de confiabilidad y validez en distintos contextos forenses, así como con poblaciones femeninas y más actualmente se ha adaptado con poblaciones adolescentes (Andrés Pueyo \& Echeburúa, 2010; Hare, 1991). En Colombia se realizó la aplicación del PCR-L en el estudio de Ruiz, (2006) a una muestra de 72 hombres quienes ingresaron a un centro carcelario en Bogotá, con edades entre los 17 y los 51 años, en el cual se encontraron índices de fiabilidad interna y validez de constructo adecuados.

Otros de los instrumentos utilizados con frecuencia en la evaluación de los adultos es el MMPI-2 (Inventario Multifásico de Personalidad de Minnesota) que evalúa la personalidad normal y patológica, seguido por el inventario Personality Assessment Inventory (PAI) cuya funcionalidad es la 
evaluación de la personalidad en casos legales que implican maltrato psicológico. Por último, el Millon Clinical Multiaxial Inventory (MCMI-III) proporciona información acerca de dificultades emocionales e interpersonales. En este orden de ideas, se puede decir que hay un uso predominante del MMPI-2, de hecho, Archer et al., (2006) dentro de su investigación afirman que los instrumentos tradicionales para la evaluación clínica como éste y el test de Rorschach desempeñan un papel fundamental para ser considerados como instrumentos de evaluación forense, mientras otros autores afirmar que por surgir en el contexto clínico son de interés limitado en el entorno forense, a pesar de su gran utilización (Hernández, 2002; Vázquez-Mezquita, 2005 citados por Echeburúa et al., 2011).

Otras técnicas de evaluación sobresalientes en la psicología clínica y aplicables en el campo forense son el WAIS-IV (Wechsler Adult Intelligence Scale) ya que da cuenta del funcionamiento cognitivo del sujeto, evalúa el Coeficiente Intelectual $(\mathrm{Cl})$ y comprende cuatro índices, que son, la comprensión verbal, la memoria de trabajo, el razonamiento perceptual, la velocidad de procesamiento, y una puntuación global del $\mathrm{Cl}$. De igual forma, pero para evaluación de niños se encuentra el WISC-IV (Wechsler Intelligence Scale for children-IV) el cual cuenta con la misma estructura del WAIS-IV, pero con subpruebas adaptadas a la población infantil. En cuanto a la evaluación neuropsicológica se utiliza el Luria- DNA el cual incluye nueve tests distribuidos en cinco áreas diferentes: viso espacial, lenguaje oral, memoria, inteligencia y atención. Por último, está el test de Barcelona realizado por Peña-Casanova, (1990, citado por Sierra et al, 2006) el cual proporciona información sobre las variables de orientación, atención y concentración, lenguaje (oral, escritura y lectura), calculo, memoria y solución de problemas.

En el ámbito de la violencia de pareja, se cuenta con el S.A.R.A. (Spousal Assault Risk Assessment) en el Manual for the Spousal Assault Risk Assessment Guide, que tiene como objetivo valorar el riesgo de violencia conyugal, está conformada por 20 factores de riesgo, de los cuales los primeros 10 valoran el riesgo de violencia en general y los segundos 10 la violencia hacia la pareja. Puello y López (2005) (citados por Andrés-Pueyo \& Echeburúa, 2010) rea- lizaron la adaptación de la prueba para los países de habla hispana pues se convirtió en prueba importante en programas con hombres maltratadores de pareja. En cuanto a violencia en adolescentes, se encuentra el VRAG cuyo propósito es evaluar el riesgo de violencia física, sexual y de amenazas graves en pacientes mentales y delincuentes jóvenes entre 14 y 18 años (Andrés-Pueyo \& Echeburúa, 2010).

Por otro lado, Alcázar, Verdejo y Bouso, (2008) proponen otros instrumentos psicológicos como el GHQ-28 -Cuestionario de Salud General De Golberg-, éste identifica la existencia de posibles problemas psicopatológicos o de salud en general de la persona durante las últimas semanas; el cuestionario de Autoevaluación Ansiedad Estado/Rasgo (STAl), que como su nombre lo indica evalúa la ansiedad-rasgo y ansiedad-estado; el inventario de Beck (BDI), que realiza la evaluación de sintomatología depresiva; Escala de riesgo de violencia de Plutchik-RV; Escala de impulsividad de Plutchik; Cuestionario de agresión ( $\mathrm{AQ}$ ), este evalúa agresividad, ira y hostilidad; escala de evitación y ansiedad social; el Test de Exploración de Alcoholismo de Michigan (TEAM); el Cuestionario de Fantasías Sexuales de Wilson; y la Escala de actitud hacia la mujer.

Así pues, para la realización del informe psicológico se debe, en primera medida, aplicar los instrumentos seleccionados para recabar información, formular hipótesis y en segunda instancia se debe analizar la información producto de la evaluación y contrastarla con otras técnicas aplicadas, en este punto se debe resaltar que los constructos psicológicos medidos en una persona deben estar ligados al caso en particular y apoyar la toma de decisiones sobre constructos legales (Grisso, 2010; Jiménez \& Sánchez, 2002; Saborío, 2005).

En cuanto a la confiabilidad, Amar y Aristizábal (2012) agregan que el perito debe tener en cuenta que el uso de instrumentos con coeficientes de confiabilidad menores a 0.8 no son recomendados en el campo forense, ya que se puede presentar un exceso de error en la varianza y limitaciones en la validez del constructo.

Ahora bien, el test o el instrumento de evaluación debe poseer un método estándar de aplicación, como señala Groth-Marnat (1999, citado por Saborío, 
2005) el instrumento debe incluir instrucciones que permita a los diferentes evaluadores aplicarlo de manera estructurada. Esto aporta en gran medida a disminuir los márgenes de error durante la aplicación. Por otra parte, es importante que la evaluación se realice en un ambiente de empatía y respeto, en donde se le explique al evaluado el objetivo de evaluación, el procedimiento, cómo se utilizaran los resultados, entre otros (Weinstein \& Weinstein, 2010).

Otro tipo de pruebas son las proyectivas. En estas, se ha encontrado una asociada a la complejidad de las diferentes interpretaciones que le puede dar el psicólogo dependiendo de su entrenamiento en este tipo de pruebas y la claridad con la cual se le presenta a los involucrados (Botha, 2010). Al respecto, existen algunos estudios que han enfatizado en la inconveniencia por debilidad científica, rigurosidad y sistematicidad, tanto en la aplicación, como en los criterios de evaluación que usan las pruebas proyectivas, dejando un componente de subjetividad en el análisis de los resultados y, por lo tanto, inconveniencia en su uso en los estrados judiciales (Archer, 2013; Lohr, Fowler \& Lilienfeld, 2002; Weiner, Spielberger \& Norman, 2002). Sin embargo en particular, una encuesta realizada en Estados Unidos se observa que los Test de Roschach, el Sentence Completion Test y la Thematic Apperception Test, son instrumentos utilizados con frecuencia; de hecho, el Test de Roschach en ese país, algunos psicólogos lo recomiendan para determinar si los niños acusados de delitos muestran síntomas de paranoia o una psicopatología en cuanto a los bajos niveles de percepción de la realidad (Archer, BuffingtonVollum, Stredny \& Handel, 2006; Botha, 2010). Por otra parte, Witt (2010) defiende el uso de las listas de chequeo como técnica para complementar las tareas complejas dentro de los procesos de evaluación en la psicología forense; pues el uso de esta herramienta posibilita que el evaluador pueda asegurar que se haya seguido los pasos mínimos necesarios para producir un informe forense competente.

En consecuencia y como se ha indicado en estos párrafos, es necesario resaltar que la evaluación en esta área se diferencia a otras áreas en cuanto a objetivos, alcance y producto de la evaluación (Amar \& Aristizábal, 2012), y en especifico, como lo han afirmado algunos estudios (Echeburúa et al.2011), la "necesidad de elaborar instrumentos específicos de evaluación forense, con las garantías suficientes de fiabilidad y validez demandadas por este contexto de intervención, es una necesidad ineludible" (p.156); y avanzar en esto, permite consolidar el proceso de evaluación en la psicología forense, como lo está en otros campos de la psicología.

\section{Método}

\section{Tipo de Estudio.}

La metodología propuesta para esta investigación es de tipo exploratorio (Hernández, Fernández, \& Baptista, 2014) en el estudio del campo psicológico forense en Colombia.

\section{Participantes}

Se incluyó un total de 40 psicólogos forenses a nivel nacional, el tipo de muestreo utilizado fue el probabilístico intencional pues los participantes fueron seleccionados por tener como características en común que fueran psicólogos forenses que trabajen como peritos en Colombia, de los cuales contestaron el instrumento 16 personas.

\section{Instrumento}

Se construyó una entrevista estructurada compuesta de 37 preguntas, divididas en las siguientes áreas: a) Datos generales del perito, b) Actividad del perito, c) Procesos y técnicas de evaluación d) Consideraciones éticas de la práctica forense. Adicionalmente, en este instrumento se realizaron preguntas abiertas, de selección múltiple y cerrados cerradas con respuesta complementarias.

\section{Procedimiento}

El estudio inició con la revisión teórica del área de la psicología forense, los problemas éticos que se podían presentar, los diferentes campos del derecho donde interviene y los instrumentos que se utilizan, para soportar la creación de la entrevista usada para la recolección de la información. Luego con la 
conceptualización que permitió la revisión teórica, se elaboró el instrumento y para su aplicación. Se contactaron inicialmente por correo electrónico un total de 40 psicólogos forenses, participando finalmente en el estudio 16 de ellos, a quienes fue aplicado de manera presencial. Posteriormente se ajustó el formato especificando los ítems cualitativos y cuantitativos de cada una de las entrevistas y se tabularon los resultados para un posterior análisis descriptivo de los mismos.

\section{Resultados}

La muestra elegida para la presente investigación estuvo conformada por 13 mujeres (81.2\%) y 3 hombres (21.4\%), con edades comprendidas entre los 26 y 52 años. El $42 \%$ de los peritos se encontraban laborando en las principales ciudades de Colombia y el $58 \%$ se encontraron en Bogotá. Con respecto a la formación académica, presentó un porcentaje mayor el nivel de maestría (68.7\%) seguido por una especialización (25\%). En cuanto a la experiencia laboral, un $62.5 \%$ de los peritos manifiestan tener experticia y dedicación en la docencia. En este sentido, un $56.2 \%$ de los peritos se encontraban laborando en una actividad distinta a las indagadas, los datos arrojaron que se realizaron dentro de un rango de 30 a 1000 peritajes y sólo han sido llevados a juicio entre 6 y 100 peritajes.
Por otro lado, se observa que la mayoría de los participantes perciben que no hay un posicionamiento fuerte de los psicólogos jurídicos en Colombia (56\%). Asimismo, se evidenció que los psicólogos forenses realizan su práctica principalmente en el ámbito penal (81\%), el civil (25\%) y el familiar (25\%). Se encontró también, que al momento de desarrollar el informe los psicólogos se inclinaban en su mayor parte por el informe pericial (94\%) en lugar del contra informe (19\%); aunque en ocasiones algunos de los psicólogos entrevistados habían realizado los dos tipos de informe y por esta razón no se excluyeron los datos. Se evidenció que la principal fuente de solicitud de peritajes fue por parte de la defensa ( $75 \%$ y se tuvo en cuenta también que algunos de los psicólogos consideraron que hay más de una fuente de solicitud (véase tabla 1).

Nota: En las categorías de campo del derecho y rol del psicólogo alguno de los 16 participantes no respondieron nada, algunos campos quedaban en cero y en otras categorías no son excluyente.

Por otra parte, se puede observar la participación de los peritos en distintos casos. En este sentido, los resultados arrojan que la categoría con mayor puntuación fue delitos sexuales en niños con una puntuación de $81.2 \%$ y en último lugar divorcio $(0.0 \%)$, como se evidencia en la tabla 2. 

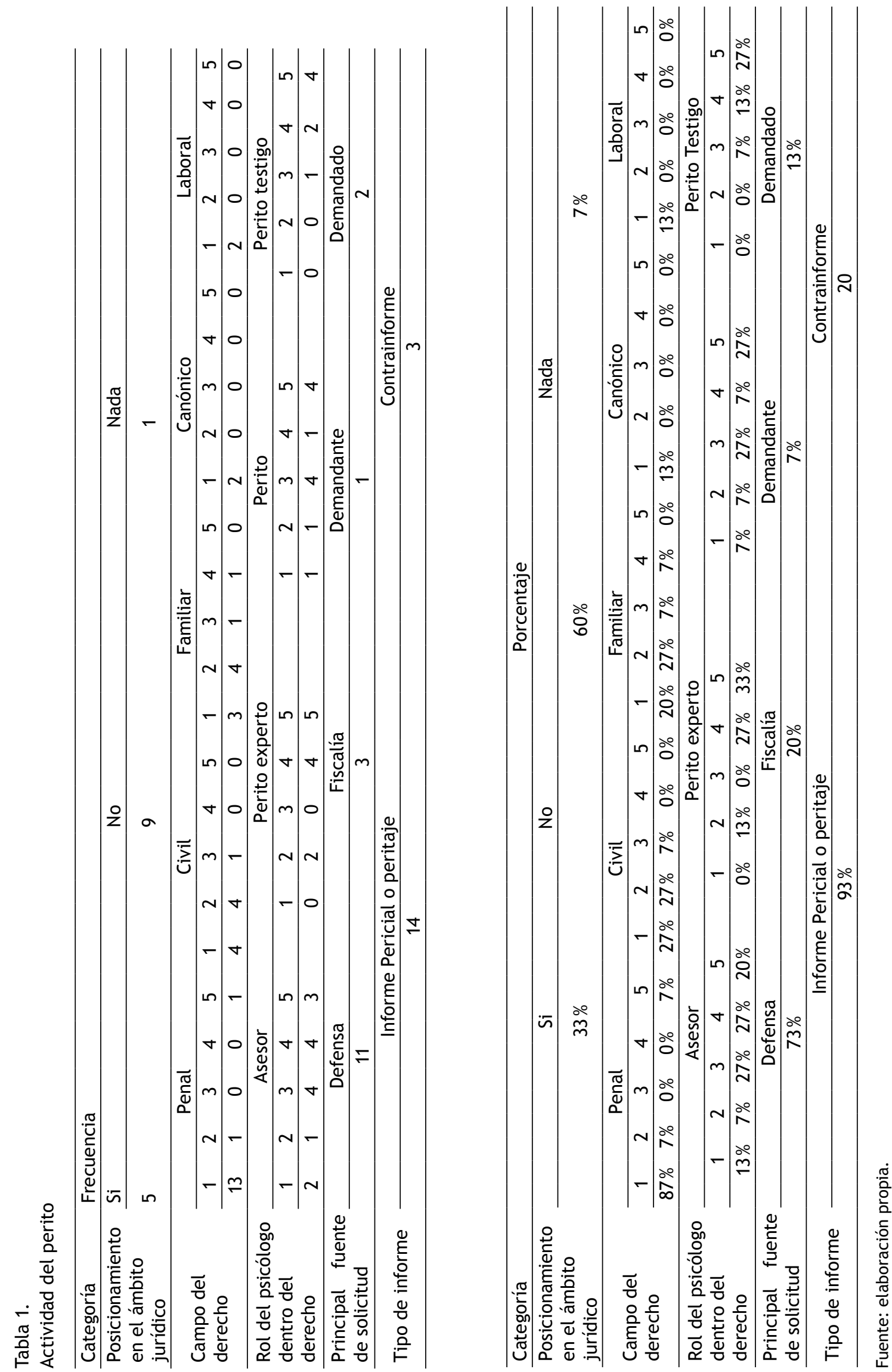
Tabla 2.

Participación de peritos.

\begin{tabular}{|c|c|c|c|c|c|c|c|c|}
\hline \multirow{2}{*}{ Categoría } & \multicolumn{4}{|c|}{ Frecuencia } & \multicolumn{4}{|c|}{ Porcentaje } \\
\hline & Alto & Medio & Bajo & Ninguno & Alto & Medio & Bajo & Ninguno \\
\hline Delito sexual en niño & 13 & 2 & 1 & 0 & 81.2 & 12.5 & 6.2 & 0.0 \\
\hline Delito sexual en adultos & 1 & 3 & 5 & 3 & 6.2 & 18.7 & 31.2 & 18.7 \\
\hline Violencia en parejas & 1 & 5 & 5 & 2 & 6.2 & 31.2 & 31.2 & 12.5 \\
\hline Homicidios & 2 & 3 & 2 & 4 & 12.5 & 18.7 & 12.5 & 25 \\
\hline Inimputabilidad & 0 & 3 & 2 & 3 & 0.0 & 18.7 & 12.5 & 18.7 \\
\hline Guarda y custodia de hijo & 1 & 2 & 6 & 3 & 6.2 & 12.5 & 37.5 & 18.7 \\
\hline Interdicción en derechos & 0 & 1 & 3 & 5 & 0.0 & 6.2 & 18.7 & 31.2 \\
\hline Evaluación de testigos & 1 & 0 & 4 & 3 & 6.2 & 0.0 & 25 & 18.7 \\
\hline $\begin{array}{l}\text { Evaluación de síndrome de } \\
\text { alienación parental }\end{array}$ & 1 & 4 & 2 & 5 & 6.2 & 25 & 12.5 & 31.2 \\
\hline Regulación de visitas & 1 & 3 & 1 & 6 & 6.2 & 18.7 & 6.2 & 37.5 \\
\hline Divorcios & 0 & 0 & 0 & 4 & 0.0 & 0.0 & 0.0 & 25 \\
\hline Adopción & 1 & 2 & 2 & 7 & 6.2 & 12.5 & 12.5 & 43.7 \\
\hline Incidentes de reparación & 2 & 1 & 3 & 4 & 12.5 & 6.2 & 18.7 & 25 \\
\hline Nulidad de matrimonio & 0 & 1 & 1 & 6 & 0,0 & 6.2 & 6.2 & 37.5 \\
\hline $\begin{array}{l}\text { Responsabilidad } \\
\text { penal juvenil }\end{array}$ & 1 & 1 & 4 & 5 & 6.2 & 6.2 & 25 & 31.2 \\
\hline Responsabilidad criminal & 0 & 3 & 0 & 5 & 0.0 & 18.7 & 0.0 & 31.2 \\
\hline Secuelas psicológicas & 4 & 3 & 3 & 3 & 25 & 18.7 & 18.7 & 18.7 \\
\hline Juego patológico & 0 & 0 & 1 & 5 & 0.0 & 0.0 & 6.2 & 31.2 \\
\hline $\begin{array}{l}\text { Determinación de } \\
\text { incapacidad permanente o } \\
\text { absoluta }\end{array}$ & 0 & 3 & 1 & 4 & 0.0 & 18.7 & 6.2 & 25 \\
\hline $\begin{array}{l}\text { Procedimiento de } \\
\text { incapacitación }\end{array}$ & 0 & 0 & 0 & 8 & 0.0 & 0.0 & 0.0 & 50 \\
\hline Mobbing & 0 & 1 & 0 & 7 & 0.0 & 6.2 & 0.0 & 43.7 \\
\hline Bullying & 0 & 0 & 3 & 7 & 0.0 & 0.0 & 18.7 & 43.7 \\
\hline Autopsia psicológica & 0 & 0 & 4 & 5 & 0.0 & 0.0 & 25 & 31.2 \\
\hline Capacidad para contratar & 0 & 0 & 0 & 6 & 0.0 & 0,0 & 0.0 & 37.5 \\
\hline Impugnación de testamento & 0 & 0 & 4 & 5 & 0.0 & 0.0 & 25 & 31.2 \\
\hline $\begin{array}{l}\text { Alteración psicopatológica } \\
\text { con incapacidad laboral }\end{array}$ & 0 & 3 & 0 & 5 & 0.0 & 18.7 & 0.0 & 31.2 \\
\hline
\end{tabular}

Fuente: elaboración propia.

Nota. Los datos fueron tomados en una muestra de 16 peritos, de esta misma cantidad fueron calculadas las frecuencias y los porcentajes. 
En la tabla 3 se encuentra la población objetivo a los cuales se les realizó el proceso de evaluación. Los resultados muestran que la categoría víctimas es la más frecuente (81.2\%).

Tabla 3.

Población de peritados

\begin{tabular}{lcc}
\hline Categoría & Frecuencia & Porcentaje \\
\hline Víctima & 13 & 81.2 \\
Víctima mujer & 10 & 62.5 \\
Víctima hombre & 3 & 18.7 \\
Agresor mujer & 3 & 18.7 \\
Agresor hombre & 9 & 56.2 \\
Demandante & 9 & 56.2 \\
Demandado & 1 & 6.2 \\
Familia víctima & 4 & 25 \\
Madre & 12 & 75 \\
Padre & 12 & 75 \\
Hijos & 11 & 68.7 \\
Testigo & 5 & 31.2 \\
\hline
\end{tabular}

Fuente: elaboración propia.

Tabla 4.

Procesos y técnicas de evaluación
En cuanto a las técnicas de evaluación que los psicólogos forenses más utilizaron (algunos usan más de una), se muestra en la tabla 4, correspondiendo a los protocolos (62.5\%), la observación y la entrevista clínica estructurada (50\%), la lista de chequeo (43.8\%), las pruebas clínicas $(43.8 \%)$ y la prueba forense $(43.8 \%)$.

Por otro lado, en la tabla 5 se describen los criterios más relevantes para seleccionar un instrumento de evaluación, encontrando: que tuviera información relevante sobre lo que se quiere indagar $(81.3 \%)$; que pudiera ser respondida por el peritado (50\%), sencillez y practicidad del instrumento (43.8) y que el perito conociera los procedimientos de aplicación y calificación (43.8).

\begin{tabular}{lcccccccccc}
\hline & \multicolumn{1}{c}{ Frecuencia } & \multicolumn{7}{c}{ Porcentaje \% } \\
\hline Técnicas & $\mathbf{5}$ & $\mathbf{4}$ & $\mathbf{3}$ & $\mathbf{2}$ & $\mathbf{1}$ & $\mathbf{5}$ & $\mathbf{4}$ & $\mathbf{3}$ & $\mathbf{2}$ & $\mathbf{1}$ \\
Entrevista clínica estructurada & $\mathbf{8}$ & 2 & 0 & 1 & 0 & 50.0 & 12.5 & 0.0 & 6.3 & 0.0 \\
Pruebas clínicas & 7 & 4 & 2 & 0 & 0 & 43.8 & 25.0 & 12.5 & 0.0 & 0.0 \\
Entrevista cognitiva & 4 & 2 & 1 & 1 & 0 & 25.0 & 12.5 & 6.3 & 6.3 & 0.0 \\
Prueba forense & 7 & 4 & 1 & 0 & 2 & 43.8 & 25.0 & 6.3 & 0.0 & 12.5 \\
Protocolo & 10 & 4 & 0 & 1 & 0 & 62.5 & 25.0 & 0.0 & 6.3 & 0.0 \\
Cuestionario & 4 & 2 & 0 & 2 & 0 & 25.0 & 12.5 & 0.0 & 12.5 & 0.0 \\
Observación & 8 & 0 & 1 & 1 & 0 & 50.0 & 0.0 & 6.3 & 6.3 & 0.0 \\
Perfiles criminales & 4 & 3 & 0 & 1 & 2 & 25.0 & 18.8 & 0.0 & 6.3 & 12.5 \\
Autopsia psicológica & 6 & 0 & 1 & 2 & 3 & 37.5 & 0.0 & 6.3 & 2.5 & 8.8 \\
Lista de chequeo & 7 & 5 & 0 & 0 & 0 & 43.8 & 31.3 & 0.0 & 0.0 & 0.0 \\
\hline Otras & 6 & 0 & 0 & 0 & 0 & 37.5 & 0.0 & 0.0 & 0.0 & 0.0 \\
\hline
\end{tabular}

Fuente: elaboración propia. 
Tabla 5.

Criterio de selección de una prueba.

\begin{tabular}{lcccccccccc}
\hline Criterio & \multicolumn{9}{c}{ Frecuencia } & \multicolumn{7}{c}{ Porcentaje - } \\
\hline & $\mathbf{5}$ & $\mathbf{4}$ & $\mathbf{3}$ & $\mathbf{2}$ & $\mathbf{1}$ & $\mathbf{5}$ & $\mathbf{4}$ & $\mathbf{3}$ & $\mathbf{2}$ & $\mathbf{1}$ \\
\hline Información Relevante & 13 & 2 & 0 & 1 & 0 & 81.3 & 12.5 & 0.0 & 6.3 & 0.0 \\
Poder responderla el peritado & 8 & 2 & 1 & 0 & 0 & 50 & 12.5 & 6.3 & 0.0 & 0.0 \\
Saberla administrar y calificar & 7 & 3 & 1 & 0 & 1 & 43.8 & 18.8 & 6.3 & 0.0 & 6.3 \\
Fiabilidad & 6 & 4 & 6 & 0 & 0 & 37.5 & 25.0 & 37.5 & 0.0 & 0.0 \\
Validez & 5 & 3 & 5 & 1 & 0 & 31.3 & 18.8 & 31.3 & 6.3 & 0.0 \\
Normativización & 3 & 2 & 6 & 0 & 0 & 18.8 & 12.5 & 37.5 & 0.0 & 0.0 \\
Adecuación al juicio & 3 & 2 & 4 & 1 & 1 & 18.8 & 12.5 & 25 & 6.3 & 6.3 \\
Sencillez y practicidad & 7 & 1 & 2 & 1 & 1 & 43.8 & 6.3 & 12.5 & 6.3 & 6.3 \\
\hline Otras & 2 & 0 & 0 & 0 & 0 & 12.5 & 0.0 & 0.0 & 0.0 & 0.0 \\
\hline
\end{tabular}

Fuente: elaboración propia.

En cuanto a los procedimiento, criterios y técnicas en la evaluación psicológica forense, se encontró que el $\mathbf{8 7 . 5 \%}$ de la muestra afirma que existen diferencias en el procedimiento de evaluación de las víctimas y victimarios de diferentes delitos. Por otra parte, el $81.3 \%$ indicó que tiene un modelo particular de protocolo para la presentación de informe o peritaje. Mientras, menos de la mitad de la población afirmó que para todos los casos la estructura del informe pericial no es la misma (43.8\%). Se encontró que el $81.3 \%$ de los encuestados dice que es relevante en los informes psicológicos forenses, explicar y sustentar científicamente las técnicas y métodos que se usaron. De acuerdo con esto, se encontró que $93.8 \%$ del total de la muestra agregan al final del informe la bibliografía consultada para sustentar sus resultados. Por último, relacionado con el proceso de evaluación, se encontró que todos hacen uso de grabaciones y transcripciones en el proceso de evaluación y el $87.5 \%$ inicia la evaluación de un peritado con la lectura del expediente.

Con respecto a los criterios para la selección de las técnicas de evaluación, el $93.8 \%$ indicó que el criterio más importante fue el objetivo del peritaje, seguido de las características del sujeto peritado (81.3) y menos importante el número de sujetos a evaluar (12.5\%)

Al preguntar acerca de los inconvenientes más comunes en una evaluación psicológica, se encontró que como el inconveniente que más se presenta (62.5\%), existencia de pocas pruebas validadas en nuestro país. En segundo lugar, menos de la mitad de la muestra (43.8\%) identificó como inconveniente que no hay instrumentos desarrollados exclusivamente en el área de la psicología forense, y en este mismo sentido el $31.3 \%$ de los participantes indicaron que las pruebas existentes tienen finalidad clínica. Finalmente, otros criterios a destacar es el limitado tiempo para aplicar las pruebas (37.5\%) y la detección de la simulación del peritado (31.3\%).

Con respecto a las pruebas específicamente se observó que las cinco mejores pruebas para realizar peritajes, según los participantes, fueron el MMPI-2 (56\%), MCMI-III (50\%), Cuestionario de Ansiedad Estado- Rasgo (STAI) (44\%), Inventario de Depresión de Beck (44\%) y Cuestionario de Salud General de Golberg ( $83 \%$ ) y en donde más de la mitad de los encuestados aseguraban una fiabilidad del $50 \%$ o más.

Relacionado a esto, en la tabla 6 se muestra que los psicólogos forenses, utilizan con mayor frecuencia la prueba MMPI-2, seguida de las pruebas ansiedad de Hamilton, ansiedad de Beck y cuestionario de fantasías sexuales de Wilson. Por otro lado, se encontró que las pruebas menos utilizadas por este tipo de profesionales son HTP, PAI, EPV-R con un porcentaje de $(6.25 \%)$, es decir solo 1 perito del total de la muestra. 
Tabla 6.

Pruebas utilizadas por los peritos

\begin{tabular}{|c|c|c|}
\hline Categorías & Frecuencia & Porcentaje \\
\hline El Raben & 7 & 43.74 \\
\hline WAIS & 10 & 62.5 \\
\hline WISC R & 6 & 37.5 \\
\hline Figura Humana & 2 & 12.5 \\
\hline Bender & 4 & 25 \\
\hline $16 \mathrm{PF}$ & 10 & 62.5 \\
\hline HTP & 1 & 6.25 \\
\hline MMPI I & 9 & 56.25 \\
\hline MMPI II & 13 & 81.25 \\
\hline EPQ & 4 & 25 \\
\hline EPQ J & 3 & 18.75 \\
\hline PPG IPG & 2 & 12.5 \\
\hline MCMI II & 10 & 62.5 \\
\hline STAI & 10 & 62.5 \\
\hline ISRA & 3 & 18.75 \\
\hline EL BDI & 8 & 50 \\
\hline STAXI & 7 & 43.75 \\
\hline Ansiedad De Hamilton & 12 & 75 \\
\hline Ansiedad Beck & 12 & 75 \\
\hline Zung & 5 & 31.25 \\
\hline Depresión Beck & 11 & 68.75 \\
\hline Test de Barcelona & 5 & 31.25 \\
\hline DNA de Luria & 7 & 43.75 \\
\hline CUMANIN & 2 & 12.5 \\
\hline $\begin{array}{l}\text { Escala de Satisfacción Familiar por } \\
\text { Adjetivos }\end{array}$ & 9 & 56.25 \\
\hline Autoconcepto Forma 5 & 3 & 18.75 \\
\hline Depresión de Beck & 10 & 62.5 \\
\hline STAI & 9 & 56.25 \\
\hline STAXI 2 & 3 & 18.75 \\
\hline CAS 8 ANSIEDAD INFANTIL & 7 & 43.75 \\
\hline CID (DEPRESION NIÑOS) & 9 & 56.25 \\
\hline $\begin{array}{l}\text { Cuestionario de Fantasías Sexuales } \\
\text { de Wilson }\end{array}$ & 12 & 75 \\
\hline SVR 20 & 10 & 62.5 \\
\hline HCR 20 & 10 & 62.5 \\
\hline PAI & 1 & 6.25 \\
\hline RRASOR & 2 & 12.5 \\
\hline SARA & 8 & 50 \\
\hline $\begin{array}{l}\text { Escala de Actitud Hacia la Mujer Spence, } \\
\text { J.T \& Helmreich }\end{array}$ & 11 & 68.75 \\
\hline
\end{tabular}




\begin{tabular}{lcc}
\hline Categorías & Frecuencia & Porcentaje \\
\hline MILLON & 9 & 56,25 \\
EPV R & 1 & 6.25 \\
SAVRI & 2 & 12.5 \\
Cuestionario de Reacciones Emocionales & 7 & 43.75 \\
SCL 90 & 10 & 62.5 \\
Inventario de Síntomas & 7 & 43.75 \\
GHQ 28 & 6 & 37.5 \\
PPDE & 2 & 12.5 \\
NEO PI & 3 & 18.75 \\
SVA & 5 & 31.25 \\
IPDE & 6 & 37.5 \\
PCL R & 9 & 56.25 \\
Impulsividad de Plutichk & 11 & 68.75 \\
ROCHARD & 3 & 18.75 \\
Machover (Figura Humana) & 2 & 12.5 \\
Riesgo de Suicidio de Plutichk & 5 & 31.25 \\
\hline
\end{tabular}

Fuente: elaboración propia.

La tabla 7 muestra los casos donde se utilizaron con más frecuencia los procesos de evaluación. Al respecto, se identificaron los casos de delitos sexuales $(100 \%)$, violencia contra la pareja $(93.75 \%$ y la interdicción de derechos con un $31.25 \%$ (5 peritos) como el aspecto en donde menos es utilizado.

Fundamentado en los resultados arrojados surgen las siguientes categorías de análisis que permiten comprender las experiencias, formación profesional y ética de los psicólogos forenses entrevistados. Los resultados en la tabla 8 muestra que los psicólogos peritos han cursado posgrados y realizado sus pasantías en esta misma área.
Tabla 7.

Casos utilizados para la evaluación y elaboración del informe.

\begin{tabular}{lcc}
\hline Categorías & Frecuencia & Porcentaje \\
\hline Delitos sexuales & 16 & 100 \\
Violencia contra la & 15 & 93.75 \\
pareja & 10 & 62.5 \\
Cuidado de niños & 13 & 81.25 \\
Separación y divorcios & 12 & 75 \\
Delitos violentos & 5 & 31.25 \\
Interdicción de & 8 & 50 \\
derechos & 10 & 62.5 \\
Inimputabilidad & 9 & 56.25 \\
Incapacidad laboral & \\
Mobbing &
\end{tabular}

Fuente: elaboración propia. 
Tabla 8.

Capacitación adicional y respaldo de entidades diferente a las universitarias para su ejercicio como psicólogo forense.

\begin{tabular}{lccccc}
\hline Programas & Frecuencia & Porcentajes & Respaldos & Frecuencia & Peritaje \\
\hline Pasantías & 2 & 13 & Colpsic & 6 & 38 \\
Diplomados & 1 & 6 & Defensoría del pueblo & 1 & 6 \\
Posgrado & 6 & 38 & Colpsic y otras asaciones & 1 & 6 \\
$\begin{array}{l}\text { Posgrado y } \\
\text { pasantía }\end{array}$ & 4 & 25 & $\begin{array}{c}\text { Colpsic y laboratorio de Psicología } \\
\text { jurídica de la U. Nacional }\end{array}$ & 1 & 6 \\
$\begin{array}{l}\text { Diplomado y } \\
\text { Otros Cursos }\end{array}$ & 1 & 6 & Ninguno & 7 & 44 \\
Curso & 1 & 6 & & & \\
No informa & 1 & 6 & & & \\
\hline
\end{tabular}

Fuente: elaboración propia.

Nota. Colegio Colombiano de psicólogos COLPSIC.

Por otra parte, en la tabla 9 se muestran una serie de categorías en la que se da un análisis de contenido de las preguntas abiertas

La tabla 10 presenta las categorías y las subcategorizas de análisis del contenido, realizado a las respuestas de los psicólogos forenses en relación con el reconocimiento y posicionamiento de los psicólogos en el país. La otra categoría corresponde a las circunstancias que determinan la selección de las pruebas para evaluar las víctimas y victimarios. Por último, las consideraciones éticas a tener en cuenta para una adecuada praxis del psicólogo, se encontraron tres sub-categorías, en cuanto al primera se encuentran acciones propias para un efectivo peritaje, como es la planeación, aplicación de las pruebas pertinentes; en lo referente a perito, condiciones éticas relacionadas con la veracidad en generar de la información y el respeto por las personas involucradas en el proceso y en la formación relacionadas con la actualización permanente y siempre la búsqueda del respaldo en las avance científicos.

Tabla 9.

Categorías encontradas en relación con la actividad del perito.

\section{Conceptualizaciones}

\begin{tabular}{|c|c|}
\hline 1 & $\begin{array}{l}\text { Actualización normativa: surgimiento de nuevas normas leyes que permiten visibilizar el rol del psicólogo } \\
\text { forense }\end{array}$ \\
\hline 2 & $\begin{array}{l}\text { Desconocimiento del rol del psicólogo forense: falta de información de entidades y profesionales del de- } \\
\text { recho acerca del conocimiento, competencias de los psicólogos forenses y el aporte de éstos a la adminis- } \\
\text { tración de justicia. }\end{array}$ \\
\hline 3 & $\begin{array}{l}\text { Competencia: capacidad de un individuo a actuar de acuerdo con su formación la expectativa que de su } \\
\text { rol se tiene. (analizar, conocer, ser ético, ser responsable sintetizar, trabajar con otros profesionales, tener } \\
\text { pasión, competencias comunicativas orales) }\end{array}$ \\
\hline 4 & $\begin{array}{l}\text { Formación: cumplimiento de programas académicos que contribuyen a la formación y la calificación de su } \\
\text { experticia. (pruebas, psicometría y medición, derecho, legislación colombiana, código deontológico.) }\end{array}$ \\
\hline 5 & $\begin{array}{l}\text { Circunstancias de elección de pruebas: situaciones de los sujetos, del delito, y de las consecuencias psico- } \\
\text { lógicas que determinan la selección y aplicación de instrumentos para las víctimas y los victimarios. }\end{array}$ \\
\hline 6 & $\begin{array}{l}\text { Condiciones éticas: "conocimiento, valores y actitudes que se enmarcan en los principios éticos, morales } \\
\text { y deontológicos de los psicólogos en general y jurídicos en particular" (Perfiles por competencias del } \\
\text { profesional en psicología, 2013). Se relacionan con proceso llevado por el perito, del perito mismo y de } \\
\text { formación y actualización de este y que su incumplimiento lleva a sanciones. }\end{array}$ \\
\hline
\end{tabular}

Fuente: elaboración propia. 
Tabla 10.

Frecuencias para el Posicionamiento del psicólogo forense en el ámbito jurídico en Colombia, cualidades y consideraciones éticas para ejercer

\begin{tabular}{|c|c|c|}
\hline Categoría & Sub - categoría & Frecuencia \\
\hline Actualización normativa & & 7 \\
\hline $\begin{array}{l}\text { Desconocimiento del rol del } \\
\text { psicólogo forense }\end{array}$ & & 11 \\
\hline \multirow{2}{*}{ Cualidades de los psicólogos forenses: } & Competencia & 29 \\
\hline & Formación & 14 \\
\hline \multirow{4}{*}{$\begin{array}{l}\text { Circunstancias de elección de pruebas } \\
\text { (Víctimas) }\end{array}$} & Objetivos de la Pericia & 8 \\
\hline & $\begin{array}{l}\text { Condiciones del sujeto (edad, sexo, } \\
\text { historia, familia, nivel educativo, etc.) }\end{array}$ & 6 \\
\hline & $\begin{array}{l}\text { Condiciones de delito (violencia, tipo de } \\
\text { víctima, daño infringido) }\end{array}$ & 5 \\
\hline & Secuelas del delito & 5 \\
\hline \multirow{4}{*}{$\begin{array}{l}\text { Circunstancias de elección de pruebas } \\
\text { (victimario) }\end{array}$} & Objetivos de la Pericia & 5 \\
\hline & $\begin{array}{l}\text { Condiciones del sujeto (edad, sexo, } \\
\text { historia, familia, nivel educativo, etc.) }\end{array}$ & 11 \\
\hline & $\begin{array}{l}\text { Condiciones de delito (violencia, tipo de } \\
\text { víctima, daño infringido) }\end{array}$ & 5 \\
\hline & Secuelas del delito & 1 \\
\hline \multirow{3}{*}{ Condiciones éticas } & Del proceso & 30 \\
\hline & Del perito & 21 \\
\hline & Formación y actualización & 4 \\
\hline
\end{tabular}

Fuente: elaboración propia.

\section{Discusión}

La psicología forense hace referencia a la búsqueda, examen y presentación de pruebas psicológicas con propósitos jurídicos, resaltando que la función del psicólogo forense o perito es asesorar, ilustrar y aportar conocimientos al juez tribunal (Finol, 2006). Por ende, el presente estudio tuvo como objetivo determinar cómo se está realizando el proceso de evaluación en el campo de la Psicología Forense en Colombia.

En Colombia, los psicólogos se desempeñaron principalmente en el ámbito penal en temas de delitos sexuales en niños y en la mayoría de los casos trabajan con víctimas, en donde, realizan evaluación de los hechos con el objetivo de generar un informe para ser presentado ante un juez, según los reque- rimientos y particularidades del caso (Finol \& Piña, 2008). Por esto es imprescindible que el psicólogo forense conozca y maneje adecuadamente el Código de Procedimiento Penal.

Por otra parte, la mayoría de los psicólogos usan el informe pericial o peritaje como informe psicológico escrito, el cual debe tener una sustentación teoría correcta y límites en la opinión; este informe debe incluir las fuentes de donde se ha obtenido la información, veracidad y sinceridad tanto en su testimonio como en su informe, claridad de los roles y mantenimiento del papel de testigo experto. En este sentido Simões en 2011, menciona que el peritaje se constituye como una prueba indirecta y de carácter científico, que tiene como objeto el análisis del comportamiento humano o la personalidad y los aspectos intelectuales o volitivos de la persona. 
Dentro del estudio, la técnica más usada por los psicólogos forenses fueron los protocolos, mientras que en 2012, Molina, Arch y Jarne afirman que las técnicas de evaluación más utilizadas son la revisión documental y la entrevista individual. Además, las técnicas se tienen que sustentar científicamente, como dice Arbach y Álvarez (2008), ya que en muchos casos las pruebas incluyen en su manual el sustento teórico y estadístico que son base para las interpretaciones o contrastación de hipótesis con respecto al caso específico tratado. De igual forma, es importante el uso de grabaciones y transcripciones como un respaldo científico, como lo determina el Código de Procedimiento Penal en el que acepta la necesidad de pruebas concernientes a grabaciones de audio y vídeos como prueba del perito en la defensa, circunstancias de participación y procedimiento.

Según la teoría, los psicólogos forenses deben realizar otras funciones como el desarrollo de pruebas psicológicas con el fin de aportar información, consultoría aplicada a diferentes instancias judiciales que participan en el proceso jurídico, la respuesta a las inquietudes o dudas que exista en relación con el comportamiento humano y particularmente de las personas intervinientes, víctimas, testigos y jueces (Crespi, 1994, citado por Morales \& García 2010).

Se encontraron diferencias con respecto a la evaluación que se realiza en víctimas y victimarios ya que depende también del caso en el que estén inmersos. Adicionalmente, los inconvenientes más comunes en el proceso de evaluación fueron: a) hay pocas pruebas validadas en nuestro país, b) no se encuentran instrumentos para la utilización exclusiva en la psicología forense, c) limitado tiempo para la aplicación de las pruebas, y d) simulación del peritado. Con respecto a este último punto en 2011, Echeburúa, Muñoz, y Loinaz mencionan que la evaluación psicológica forense cuenta con algunas dificultades como lo es la involuntariedad con la que el sujeto asiste a la evaluación, los intentos por manipular la información suministrada o la influencia del proceso legal en el estado mental del sujeto.

En este estudio es importante identificar los instrumentos más utilizados por los peritos en el campo de la psicología forense. Actualmente estos son: a)
MMPI-2, b) BDI, c) Cuestionario de Fantasías Sexuales de Wilson d) Ansiedad de Hamilton. Estas pruebas fueron seleccionadas mediante un criterio personal de los peritos. En este sentido, es pertinente identificar los criterios de selección y aplicación de los instrumentos de evaluación forense en relación con el campo del derecho, problemática jurídica y cualidades del sujeto evaluado. Por consiguiente, los criterios que se tienen en cuenta con mayor frecuencia para la selección de los instrumentos son: a) el instrumento tenga información relevante sobre lo que se requiere indagar $y \mathrm{~b}$ ) que pueda ser respondida por el peritado. Sin embargo, en 2008, Arbach y Álvarez encontraron que una de las primeras condiciones para la elección de las pruebas, es que deba ser comercialmente disponible junto a un manual indicando que ha sido revisado por expertos en el área específica.

\section{Conclusiones}

El estudio por su naturaleza exploratoria y de acuerdo a la selección de la muestra de carácter intencional, no permite generalizar en la población colombiana de psicólogos y por lo tanto, los resultados del estudio brindan un panorama inicial que requiere de un estudio más amplio. Sin embargo, es posible identificar algunas percepciones y características de la praxis psicológica forense que arrojan luces de la forma en la cual se está avanzando en el país y la necesidad de continuar en el esfuerzo por hacer cada vez más visible la aplicación del rigor científico en la práctica psicológica forense.

Finalmente, la información descriptiva presentada en el estudio caracteriza las dificultades de posicionamiento, de procedimiento y éticas en relación con el ejercicio como perito. Además, un aspecto particular en el estudio corresponde a las consideraciones éticas que mencionan los profesionales al interior de su ejercicio y en las cuales existe un interfaz sensible de los principios éticos, deontológicos y morales. En otras palabras y en consonancia con Molina (2011), es posible afirmar que, por la naturaleza forense y el impacto de sus actuaciones, estos profesionales se encuentran expuestos a diferentes demandas que enmarcan problemas éticos y deontológicos 


\section{Referencias}

Alcázar, M., Verdejo, A., \& Bouso, J. (2008). El Psicólogo Forense en el Equipo Técnico de la Jurisdicción de Menores. Propuesta de Protocolo de Intervención. Anuario de Psicología Jurídica,18, 45-60.

Amar, J. \& Aristizábal, E. (2012). Psicología forense: estudio de la mente criminal. Barranquilla, Colombia: Editorial Universidad del Norte.

Amor, P., Corral, P., \& Echeburúa, E. (2003). Autoinformes y entrevistas en el ámbito de la Psicología Clínica Forense: limitaciones y nuevas perspectivas. Análisis y Modificación de Conducta, 29(126), 503-522.

Andrés-Pueyo, A., \& Echeburúa, E. (2010). Valoración del riesgo de violencia: instrumentos disponibles e indicaciones de aplicación. Psicothema, 22(3), 403-409

Archer, R. (2013). Forensic uses of clinical assessment instruments. Mahwah, NJ: Routledge.

Archer, R., Buffington-Vollum, J., Stredny, R., \& Handel, R. (2006). A survey of psychological test use patterns among forensic psychologists. Journal of Personality Assessment, 87(1), 84-89.

Arbach, K., \& Álvarez, E. (2008). Evaluación de la violencia psicológica en la pareja en el ámbito forense. Barcelona, España: Departamento de Justicia

Archer, R., Buffington, J., \& Handel, R. (2006). A survey of psychological test use patterns among, Journal of Personality Assessment, 87(1), 84-94.

Botha, E. M. (2010). Purposes for using psychological instruments in loss of income claims. Pretoria: University of South Africa.

Buela, G., Sierra, C., Carretero, H., \& de los Santos, M. (2002). Situación actual de la evaluación psicológica. Papeles del psicólogo, 1(83), 65-73.
Céspedes, R. (2002). Psicología Forense principios fundamentales. Costa Rica: Editorial Universidad Estatal de Costa Rica.

Código Penal Colombiano. (2000). Recuperado de http://www.secretariasenado.gov.co/senado/ basedoc/ley/2000/ley_0599_2000.html

Collado, J. (2009). Teoría y práctica de la investigación criminal. Instituto Universitario General Gutiérrez Mellado-UNED. Recuperado de http:// eprints.ucm.es/11021/1/psicologia_forense.pdf

Davoglio, T. \& De Lima, I. (2010). Avalição de comportamentos anti-sociais e traços psicopatas em psicología forense. Avalição Psicológica, 9(1), 111-118.

De la Calle, N., Gómez, M., Gómez, R., Muñoz, J., \& Vásquez, B. (2012). Guía de buenas prácticas para la evaluación psicológica forense del riesgo de violencia contra la mujer en las relaciones de pareja (VCMP). Madrid: Colegio Oficial de Psicólogos de Madrid.

Echeburúa, E., Muñoz, J., \& Loinaz, I. (2011) La evaluación psicológica forense frente a la evaluación clínica: propuestas y retos de futuro. International Journal of Clinical and Health Psychology, 11(1), 141-159.

Finol, M. (2006). Intervención del psicólogo forense en la administración de justicia. Capítulo Criminológico, 34(1), 99-131.

Finol, M. \& Piña, E. (2008). Intervención del psicólogo forense en la determinación de la enfermedad mental como causa de inimputabilidad. Capitulo criminológico, 36(4), 89-119.

Frías, H. (2007) ¿Que es un peritaje psicológico? México. Recuperado de http://www.peritajepsicologico.com.mx/peritaje_psicologico.asp.

Goldman, A. (s. f.). Maltrato de la mujer. Recuperado de http://www.foroaps.org/files/viole.pdf

Grisso, T. (2010). Guidance for improving forensic reports: A review of common errors. Open Access Journal of Forensic Psychology, 2, 102-115. 
Hare, R.D. (1991) The Hare psychopathy Checklist-Revised. Toronto, Canada: MultiHealth Systems.

Hernández, R., Fernández, C., \& Baptista, M. P. (2014). Metodología de la investigación $\left(6 .^{a}\right.$ ed.). México: Editorial McGraw Hill.

Hernández, E., \& Tapias A. (2010). Maltrato infantil: normatividad y Psicología Forense. Diversitas: Perspectivas en Psicología, 6(2), 389-413.

Inda, M., Lemos, S., López, A., \& Alonso J. (2005). La simulación de enfermedad física o trastorno mental. Papeles del psicólogo, 26(92), 99-108.

Jiménez, F., \& Sanchez, G. (2002). Evaluaciones psicológicas forenses: contribución de las técnicas de Minessota Millon. Amaru Ediciones.

Lin Ching Céspedes, R. (2002) Psicología forense: principios fundamentales. San José, Costa Rica: EUNED.

Lohr, J., Fowler, K., \& Lilienfeld, S. (2002). The dissemination and promotion of pseudoscience in clinical psychology: The challenge to legitimate clinical science. The Clinical Psychologist, 55, 4-10.

Masip, J. (2005). ¿Se pilla antes a un mentiroso que a un cojo? Sabiduría popular frente a conocimiento científico sobre la detección no-verbal del engaño. Papeles del psicólogo, 26(96), 78-91.

Molina, A. (2011). Conocimiento y aplicación de los principios éticos y deontológicos por parte de los psicólogos forenses expertos en el ámbito de familia. Tesis doctoral. Universidad de Barcelona. España.

Molina, A., Arch, M., \& Jarne, A. (2012). Conocimiento y aplicación de los principios éticos y deontológicos por parte de los psicólogos forenses expertos en el ámbito de familia. Anuario de psicología jurídica, 22(1), 77-93.

Morales, L., \& García, E. (2010). Psicología jurídica, quehacer y desarrollo. Diversitas: Perspectivas en Psicología, 6(2), 237-256.
Muñoz, J., \& Manzanero, A. (2011). ¿Qué es la Psicología Forense?. En: Anuario de Psicología Forense. Madrid: Plantilla Ethereal.

Natenson, S. (2007). Rol del perito psicólogo en el ámbito judicial. Psicocodebate, 8, 79-86.

Ochoa, G. (2006). Derecho civil I: personas. Caracas, Venezuela: publicaciones ucab.

Rodríguez, L. (2010). El rol del perito psicólogo en Colombia. Cultura, educación y sociedad. Revista CES, 1(1), 147-153.

Roldán, J., \& Solano, L. (2001). Departamento de Medicina Legal Organismo de Investigación Judicial poder judicial. Revista Medicina Legal, 18(1), 5-10.

Rubio, P. (2010). Victimología Forense y Derecho Penal. España: Editorial EDITUM.

Ruiz, J. (2006). Adaptación de la escala PCL-R 20 para evaluación de rasgos psicopáticos en una muestra carcelaria. Avances en medición, 4, 61-72.

Saborío, C. (2005). Estrategias de evaluación psicológica en el ámbito forense. Medicina Legal de Costa Rica, 22(1), 41-63.

Sierra, J. C., Jiménez, E. M., \& Bunce, D. (2006). Técnicas de Evaluación en psicología jurídica y forense. En J. C. Sierra, E. M. Jiménez \& G. Buela-Casal, Psicología forense: Manual de técnicas y aplicaciones. Madrid, España: Biblioteca Nueva.

Simões, (2001). Informes psicológicos en contextos forenses. En F. Jiménez Evaluación Psicológica forense (pp. 157-183). España: Amarú Ediciones.

Soria, M. (2006). Psicología jurídica un enfoque criminológico. España: Editorial Delta

Tapias-Saldaña, A. (2011). Aplicación de los instrumentos de reincidencia en violencia HCR-20 y SVR-20 en dos grupos de delincuentes colombianos. Revista Criminalidad, 53(1), 307-327. 
Vargas, N., Rodríguez, L., \& Norza, E. (2017). Psicología jurídica en Colombia: una historia en construcción. En E. Norz \& G. Egea, ConCiencia Criminal: Criminología, psicología jurídica y perfilación criminal (pp 11-30). Bogotá: Manual Moderno.

Weinstein, R. \& Weinstein, J. (2010). Culturally Competent Criminal Forensic Psychological Evaluations. 213-238. En L. F. Ramirez (Ed.),
Cultural issues in criminal defense. Juris Publishing, Inc.

Witt, P. H. (2010). Forensic report checklist. Open Access Journal of Forensic Psychology, 2, 233-240.

Weiner, I., Spielberger, Ch. and Norman, A. (2002). La psicología científica y el test de Rorschach. The Clinical Psychologist, 55, 7-12. 